\title{
THE GEOMETRY OF FLAG MANIFOLD AND HOLOMORPHIC EXTENSION OF SZEGÖ KERNELS FOR \\ $\mathrm{SU}(p, q)$
}

\author{
L. Barchini, S.G. Gindikin and H.W. Wong
}

Let $G_{0}=\mathrm{SU}(p, q), K_{0}=\mathrm{S}(\mathrm{U}(p) \times \mathrm{U}(q))$ a maximal compact subgroup, and let $G, K$ be their complexifications. Finally, let $B$ be a Borel subgroup of $G$. We define a number of algebraic functions on $G / B \times G / K$ and use them to construct a Stein extension of the Riemannian symmetric space $G_{0} / K_{0}$. These functions capture the singularities that can occur in the meromorphic extensions of the Knapp-Wallach Szegö kernels. These facts imply that all solutions of the Schmid equations extend holomorphically to the space of linear cycles.

\section{Introduction.}

A Szegö mapping is an intertwining operator from a principal series representation to a discrete series representation. Knapp and Wallach write down a number of Szegö mappings explicitly in $[\mathbf{K W}]$, in which they realize the discrete series as the solution space of the Schmid equations on a Riemannian symmetric space $Z_{0}$. It will follow as an easy consequence from our work that these solutions extend holomorphically to an universal Stein symmetric domain $Z$ containing $Z_{0}$. While this paper is strictly concerned with the Szegö mapping and its holomorphic extension, and is self-contained in this respect, we believe it is important to make explicit the rich underlying geometry. We will prove various geometric assertions made in this introduction in another paper [BGW].

It is standard in projective geometry to study duality of homogeneous spaces. In our setting, the two spaces are complex homogeneous spaces $X, Z$, where $X$ is a generalized flag manifold and $Z$ a Stein symmetric space. The same geometric data is encapsulated by a triple $(G, K, B)$, where $G$ is a connected complex reductive group, $K$ a subgroup which is the fixed point set of a complex involution, and $B$ a parabolic subgroup, so that $X=G / B, Z=G / K$. The duality is most conveniently captured by the following double fibration picture, in which $Y=G /(K \cap B)$ :

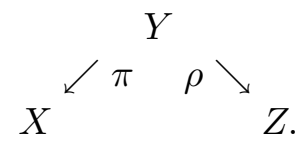


We will specialize in this paper to the following triples: $G=\mathrm{SL}(p+q, \mathbb{C})$, $B$ is a Borel subgroup, and $K$ is the subgroup $\mathrm{S}(\mathrm{L}(p) \times \mathrm{L}(q)$ ) (which is the subgroup of $\mathrm{SL}(p+q, \mathbb{C})$ consisting of matrices which have diagonal block of size $p \times p$ in the upper left corner and $q \times q$ in the lower right hand corner). We assume $p \geq q$ and let $n=p+q$. The advantage of this situation is that we can express everything in terms of objects from basic projective geometry, although the phenomenon is more subtle than that of the classical geometry.

To see what sort of interesting geometry could be done in this setting, it is instructive to recall the more elementary situation of Matineau theory of duality of complex projective spaces, see [Mar1], [Mar2], and [GH]. Let $D$ be a linearly convex domain of $\mathbb{P}_{n}$, in the sense of Martineau (i.e., through each point in $\mathbb{P}_{n} \backslash D$ is a hyperplane lying inside $\mathbb{P}_{n} \backslash D$; clearly, all convex domains, in the real sense, are linearly convex). Let $D^{*} \subseteq \mathbb{P}_{n}{ }^{*}$ consist of hyperplanes which does not intersect $\partial D$, it is called the polar set. Then there is an operator, known as the Fantapié indicator, which sets up an isomorphism between $H^{*}(\bar{D})$ (the space of analytic functionals) and $H\left(D^{*}\right)$ (the space of holomorphic sections of a line bundle). If we interpret the analytic functional as an integral operator over $\partial D$, what the Fantapié indicator does is to integrate the function $\langle\eta, z\rangle^{-1}$ ( $z$ is the variable) against a suitable integral kernel, and it then yields the value at the point in $D^{*}$ represented by $\eta$. Since the singularity of $\langle\eta, z\rangle^{-1}$ is the hyperplane defined by $\eta$, we know that we can integrate over $\partial D$ for any $\eta$ representing a point in $D^{*}$.

We would like to do the same in the more complicated setting of the flag manifold $X$. It turns out that for each point $z \in Z$, its stabilizer $G_{z} \cong K$ acts on $X$ with a unique open orbit $\mathfrak{D}(z)$. Its complement is a union $\mathfrak{O}(z)=\bigcup_{k=0}^{q} \mathfrak{O}_{k}(z)$ of the closure of exactly $q+1$ orbits $\mathfrak{O}_{k}(z)^{0}$ of codimension one. We will call $\mathfrak{D}(z)$ a determinant configuration, and the singular varieties $\mathfrak{O}_{j}(z)$ determinant varieties. From the other direction, suppose $A \subseteq X$ is any subset, define the polar of $A, \hat{A}$, to consist of those $z \in Z$ such that $\mathfrak{O}(z)$ does not intersect $A$. Obviously, $\mathfrak{O}(z)$ plays the role of hyperplane in ordinary projective geometry. However, a configuration $\mathfrak{O}(z)$ is more complicated than a hyperplane, and there are not so many "large" subsets $A$ whose polar $\hat{A}$ is non-empty. These reflect the fact that flag manifold is more complicated than projective space. To put this complexity in a slightly different way, all these reflect the rigidity of symmetric spaces with rank higher than one, of flag manifolds, or of flag domains.

It turns out that we have bihomogeneous polynomials $D_{j}(x, z)$, in terms of suitable homogeneous coordinates of $x$ on $X$ and $z$ on $Z$, such that the equation $D_{j}(, z)=0$ defines $\mathfrak{O}_{j}(z)$. We call the functions $D_{j}$ determinant functions, for they can be expressed as suitable determinants. These func- 
tions $D_{j}$ contribute to all the singularities that can occur when we extend holomorphically any Knapp-Wallach Szegö kernel. To give readers a sense of what is happening, we would like to mention that we can also write down Szegö kernels for generalized flag manifolds. The more "degenerate" the flag manifold is (i.e., if the generalized flag manifold is $G / P$, then the larger $P$ is), the less will be the number of factors involved in the singularities of the Szegö kernels. In the limiting cases of the Grassmanians $\mathrm{G}(p, p+q), \mathrm{G}(q, p+q)$, only two "determinant functions" are involved, and they can be expressed in terms of norm functions of the related Jordan algebras. The pullback via $X \rightarrow G(p, p+q)$ (or $G(q, p+q)$ ) of these two limiting determinant functions are $D_{0}$ and $D_{q}$, and occur in the Cauchy-Szegö kernels for Hermitian symmetric manifolds. The remaining $q-1$ functions are genuinely new and are particularly interesting.

The second key ingredient in Martineau's theory is the boundary of the linearly convex domain. In our context, we look for a class of "large" submanifolds whose polars are non-empty. We need a real form of $G$. In fact, we are interested only in the conjugacy (under $G$ on $G_{0}$ ) class of real forms, just as we consider the conjugacy class $\left\{G_{z}, z \in Z\right\}$ of $K$. Each member of the class acts on $X$ with a unique minimal (closed) orbit. Thus we have a class of submanifolds: The $G$ translates of the minimal $G_{0}$ orbit $X_{0}$. The polar $\hat{X}_{0}$ turns out to be a Stein extension of the real form $Z_{0}=G_{0} / K_{0} \subseteq Z$. Indeed, as complex manifolds, a connected component of $\hat{X}_{0}$ satisfies $\left(\hat{X}_{0}\right)^{0} \cong Z_{0} \times \overline{Z_{0}}$. We will see that $\hat{X}_{0}$ plays an important role in the Szegö kernels and holomorphic extension of solution of Schmid equations.

We can also understand the connected component of $\hat{X}_{0}$ from another direction. There are $\frac{(p+q) !}{p ! q !}$ open $G_{0}$ orbits on $X$, called the flag domains. It is well-known that cohomology on these flag domains realize various discrete series representations. All the flag domains share $X_{0}$ as the same "Shilov boundary". Pick any flag domain $D$. Among the minimal orbits of $K$, exactly one, $\Gamma$, lies inside $D$. The class of all $G$ translates of $\Gamma$ which stay inside $D$ is called the class of linear cycles associated to $D$. The spaces of linear cycles associated with all but two of the domains $D$ are parametrized by the a connected component of $\hat{X}_{0}$ (as can be shown by a direct computation, but this also follows from a general result proved by J. Wolf and R. Zierau). On the other hand, in [Wo1] it is proved that the space of linear cycles is always Stein, but we can prove the Stein property directly. Recall how to prove an open subset $\hat{X}_{0}$ of a Stein manifold $Z$ to be Stein, it is enough to construct barrier functions for each $z \in \partial \hat{X}_{0}$. The functions $(D(x,))^{-1}=\Pi_{j=0}^{q}\left(D_{j}\right)^{-1}$ for each $x \in X_{0}$ are what we need. Thus we see that our determinant functions give a natural way of proving the Stein property. 
A final ingredient in Martineau's theory is the Fantapié indicator. The analogue here is the holomorphic extensions of Szegö kernels. For a real semisimple Lie group $G_{0}$ with non-empty discrete series, each Knapp-Wallach Szegö mapping sends sections of a certain vector bundle on the homogeneous space $S_{0}=G_{0} / P_{0}\left(P_{0}\right.$ is a minimal parabolic subgroup $)$ to the solution space of the system of elliptic differential equations discovered in [Sc1] on the Riemannian symmetric manifold $Z_{0}$ : The Schmid equations. We would like to extend holomorphically the Knapp-Wallach Szegö kernels. In the case of $\mathrm{SU}(1,1)$, the Szegö kernel of Knapp-Wallach coincides with the Cauchy kernel for the unit disc, and its holomorphic extension is self-evident. In [Gi] is discussed how to do so in $\mathrm{SU}(2,1)$. See also $[\mathbf{K n}]$. In general, we need to "complexify" the homogeneous manifolds $S_{0}$ and $Z_{0}$. Instead of $Z_{0}$, we consider $Z$, and instead of $S_{0}$, we consider $X$ (for reasons to be explained in Section 3). The kernel function of the Szegö mapping is an analytic section, over $X_{0} \times Z_{0}$, of a certain bundle on $X \times Z$. We would like to extend this kernel holomorphically as far as possible.

We will show that we will end up with a meromorphic object $\frac{P(x, z)}{Q(x, z)}$, where $P(x, z)$ is a section of a suitable bundle and is expressible in terms of bihomogeneous polynomials, $Q(x, z)$ is a product of powers of $D_{j}$. This explains another reason why we are interested in the configurations $\mathfrak{O}(z)$ : all Szegö kernels of the Knapp-Wallach type can be extended to at least its complement.

Morally, the Szegö mapping is obtained by integrating over $X_{0}$ of the Szegö kernel. Recall the set $\hat{X}_{0}$. From what we know about the possible singularity of the holomorphic extension of the kernel, we know that the Szegö mapping will actually send a suitable section of a line bundle on $X_{0}$ to a holomorphic section of a related bundle on $\hat{X}_{0}$. Thanks to the work in $[\mathbf{K W}]$, we know that the restriction of such holomorphic sections to $Z_{0}$ give all the solutions of Schmid equations when the parameters are "far from the wall". Hence we have found an "universal" Stein extension of $Z_{0}$ over which we always have a holomorphic extension of the solutions of the Schmid equations. This is reminiscent of the classical result of Aronshain about the universal extension of solutions of Laplace equations. Later, this result is generalized by Martineau and Kiselman to a system with constant coefficients. We have here an universal domain for the extensions of the solutions of elliptic equations with variable, instead of constant, coefficients.

Here is a brief summary of the paper. In Section 2 we define $q+1$ determinant functions in order to construct a Stein extension for the Riemannian symmetric space $G_{0} / K_{0}$. We shall summarize the construction of the KnappWallach Szegö mappings in Section 3. We go to the nuts and bolts of the computation, when $G_{0}=\mathrm{SU}(p, q)$, in Sections 4 and 5 , and the upshot of the 
computations is that the singularities of the Knapp-Wallach Szegö kernels can be be described as the zero set of some of the determinant functions. From this it follows that the solution of Schmid equations can always be extended to a universal domain. Some of the results stated in Section 5 are proved in Section 6.

\section{Determinant Functions.}

We will define certain functions called the determinant functions, and we will use them to define a Stein manifold. The determinant functions will be seen to capture the singularities of the meromorphic extension of Szegö kernels and the solution of the Schmid equations will be seen to extend holomorphically to the Stein manifold.

We start with certain notations which we use throughout the paper. We start with the obvious geometric interpretations of $X$ and $Z$. The space $X$ is the set of all sequence $\left(V_{i} ; 1 \leq i \leq n=p+q\right)$ of subspaces of $\mathbb{C}^{n}$, where $\operatorname{dim} V_{i}=i$ and $V_{i} \subseteq V_{j}$ if $i \leq j$. We can represent a point of $X$ by $\omega=\left(\omega_{i}, 1 \leq i \leq p+q\right)$, where $\omega_{i} \in \Lambda^{i} \mathbb{C}^{p+q}$. Strictly speaking, we should look at the $n$-tuple $\left(\tilde{\omega}_{i}\right) \in \Pi_{i=1}^{n} \mathbb{P}\left(\Lambda^{i} \mathbb{C}^{n}\right)$, where $\tilde{\omega}_{i}$ is the projective point in $\mathbb{P}\left(\Lambda^{i} \mathbb{C}^{n}\right)$ represented by $\omega_{i}$. The space $Z$ consists of pairs $\left(L_{p}, L_{q}^{\prime}\right)$ of disjoint subspaces of dimensions $p$ and $q$. We can likewise think of $L_{p}, L_{q}^{\prime}$, as in the case of $\omega_{j}$, as two elements in $\Lambda^{\bullet} \mathbb{C}^{n}$. (Of course, there are various algebraic conditions to be satisfied by $\omega_{i}$ and $L_{p}, L_{q}^{\prime}$, but we will not list them here. We only want to point out that $L_{p} \wedge L_{q}^{\prime} \neq 0$.) Sometimes we find it convenient to use an ordered basis $\left(v_{i}, 1 \leq i \leq n\right)$ to represent a flag, by putting $\omega_{i}=v_{1} \wedge \ldots \wedge v_{i}$.

For the sake of simplicity, we find the following notation $\doteq$ useful. It means that the two sides are equal up to a non-zero constant multiple.

We introduce two more pieces of notations, for convenience's sake. On $\Lambda \bullet \mathbb{C}^{p+q}$ we have a "star" operator. Suppose we have an ordered basis $\left(v_{i}, 1 \leq\right.$ $i \leq p+q)$ for $\mathbb{C}^{p+q}$, then $* v_{k_{1}} \wedge \ldots \wedge v_{k_{i}}:=\epsilon v_{k_{i+1}} \wedge \ldots \wedge v_{k_{p+q}}$. Here $\epsilon$ is +1 or -1 according to whether $\left(k_{1}, \ldots, k_{p+q}\right)$ is an even or odd permutation of $(1, \ldots, p+q)$. Now suppose $u \in \Lambda^{a} \mathbb{C}^{p+q}, v \in \Lambda^{b} \mathbb{C}^{p+q}$, and suppose $a+b \geq$ $p+q$, define $u \sqcap v:=\iota(* u) v$. Here $\iota()$ means the interior product. Note that $u \sqcap v \doteq v \sqcap u$. Geometrically, if $u, v$ represent subspaces of dimensions $a$ and $b$, then $u \sqcap v \neq 0$ iff the subspaces represented by $u, v$ intersect transversally and in that case $u \sqcap v$ represents their intersection.

Next, for any forms $\omega, \theta$, define $\omega \sqcup \theta:=\omega \wedge \theta$. Observe that $\omega \sqcup \theta \doteq \theta \sqcup \omega$. The reason we introduce this notation is due to the following geometric interpretation. If $\omega, \theta$ represent subspaces, then $\omega \sqcup \theta \neq 0$ iff they do not intersect, and in that case $\omega \sqcup \theta$ represents their linear span. 
We will take the convention that $a \sqcup b \sqcup c:=(a \sqcup b) \sqcup c$, likewise for $a \sqcap b \sqcap c$. We will take $\left(e_{1}, \ldots, e_{p+q}\right)$ as the standard ordered basis of $\mathbb{C}^{p+q}$. We let $l_{p}=e_{1} \wedge \ldots \wedge e_{p}, l_{q}^{\prime}=e_{p+1} \wedge \ldots \wedge e_{p+q}$ be the standard $p$-plane and $q$-plane.

Given any forms $u$ of the top degree, we can identify it as a scalar $[u]$, provided we have made a choice of a "standard" top form, as we have already done.

We define $q+1$ determinant functions on $X \times Z$ as follows.

Definition 2.1. For $0 \leq j \leq q$, define $D_{j}=D_{j}\left(\omega_{1}, \ldots, \omega_{p+q} ; L_{p}, L_{q}^{\prime}\right)$ as follows.

$$
D_{j}= \begin{cases}{\left[\omega_{p} \sqcup L_{q}^{\prime}\right]} & \text { if } j=0, \\ {\left[\left(\omega_{p+q-j} \sqcap L_{p}\right) \sqcup \omega_{j} \sqcup L_{q}^{\prime}\right]} & \text { if } 1 \leq j \leq q-1, \\ {\left[\omega_{q} \sqcup L_{p}\right]} & \text { if } j=q .\end{cases}
$$

Remark 2.2. The "functions" $D_{j}$ are in fact sections of suitable line bundles on $X \times Z$. To be more precise, proceed as follows. On $Z$ are two tautological vector bundles, denoted by $V_{p}$ and $V_{q}^{\prime}$. The fiber of $V_{p}$, respectively $V_{q}^{\prime}$, over the point $\left(L_{p}, L_{q}^{\prime}\right)$ (viewing it as a pair of vector spaces) is just $L_{p}$, respectively $L_{q}^{\prime}$. Likewise, over $X$ are various tautological bundles $U_{i}$, whose fiber over a point represented by $\omega=\left(\omega_{j}\right)$ in $X$ is the vector subspace represented by $\omega_{i}$. We can form the top exterior products of all these bundles and obtain line bundles $\mathcal{L}_{Z, p}$ and $\mathcal{L}_{Z, q}^{\prime}$ on $Z$ and $\mathcal{L}_{X, i}$ on $X$. With these notations at hand, $D_{j}$ is a section of $\left(\mathcal{L}_{X, p+q-j} \otimes \mathcal{L}_{X, j}\right) \otimes\left(\mathcal{L}_{Z, p} \otimes\right.$ $\mathcal{L}_{Z, q}^{\prime}$ ) for $1 \leq j \leq q-1$, of $\mathcal{L}_{X, p} \otimes \mathcal{L}_{Z, q}^{\prime}$ for $j=0$, and of $\mathcal{L}_{X, q} \otimes \mathcal{L}_{Z, p}$ when $j=q$.

Remark 2.3. Further, these functions are pullbacks via the obvious projection $X \rightarrow Y$, where $Y$ is the generalized flag manifold consisting of subspaces $V_{1} \subseteq \ldots V_{q} \subseteq V_{p} \subseteq V_{p+1} \ldots V_{p+q-1} \subseteq V_{p+q}$, where $\operatorname{dim} V_{i}=i\left(V_{p}\right.$ is redundant if $p=q$ ). The manifold $Y$ is the complexification of the manifold $G_{0} / P_{0}$, on which the principal series in $[\mathbf{K W}]$ is realized.

Next, let $G_{0}=\mathrm{SU}(p, q)$ and $K_{0}=\mathrm{S}(\mathrm{U}(p) \times \mathrm{U}(q))$ be its maximal compact subgroup. The space $Z_{0}=G_{0} / K_{0}$ is a Hermitian symetric space. Concretely,

$$
Z_{0}=\left\{L_{p} \in G(p, p+q) \mid\langle,\rangle \text { is positive definite on } L_{p}\right\} .
$$

Here we define $\langle z, z\rangle:=\sum_{i=1}^{p}\left|z_{i}\right|^{2}-\sum_{j=p+1}^{p+q}\left|z_{j}\right|^{2}$. We shall identify the conjugate manifold $\overline{G_{0} / K_{0}}$ as

$$
\overline{Z_{0}}:=\left\{L_{q}^{\prime} \in G(q, p+q) \mid\langle,\rangle \text { is negative definite on } L_{q}^{\prime}\right\} .
$$

Hence there is the obvious identification of $Z_{0} \times \overline{Z_{0}} \subseteq Z=G / K$. 
We will from now on identify

$$
Z_{0}=\left\{\left(L_{p},\left(L_{p}\right)^{\perp}\right) \mid\langle,\rangle \text { is positive definite on } L_{p}\right\} \subseteq Z_{0} \times \overline{Z_{0}} .
$$

Here $\perp$ denotes the orthogonal complement with respect to $\langle$,$\rangle . The$ advantage of this point of view is that $Z$ is manifestly a $G_{0}$ invariant complexification (indeed Stein) extension of $Z_{0}$.

It is well known that $X$ has a unique closed $G_{0}$ orbit $X_{0}$ which is $K_{0}$ homogeneous ([Wo1]). It can be realized geometrically as follows. On $\mathbb{P}_{n-1}$ are three $G_{0}$ orbits, two of them are open (those points on which $\langle$,$\rangle are$ positive, resp. negative), the remaining one $\Sigma$ is closed. A flag $\omega=\left(\omega_{j}, 1 \leq\right.$ $j \leq n$ ) belongs to $X_{0}$ iff the following is true: $\omega_{q} \subseteq \Sigma$ (here, we view $\omega_{q}$ as a projective plane of dimension $q-1$ and $\Sigma$ a subset in $\left.\mathbb{P}_{n-1}\right)$ and $\omega_{n-1}$, when viewed as a hyperplane of $\mathbb{P}_{n-1}$, is tangent to $\Sigma$ at any point of $\omega_{q}$.

Now consider the set

Definition 2.4. Define $\hat{X}_{0} \subseteq Z$ as follows.

$$
\hat{X}_{0}:=\left\{\left(L_{p}, L_{q}^{\prime}\right) \mid D_{j}\left(\omega ; L_{p}, L_{q}^{\prime}\right) \neq 0 \text { for all } j \text { and for all } \omega \in X_{0}\right\} .
$$

Let $D:=\Pi_{j} D_{j}$, then

$$
\hat{X}_{0}=\left\{\left(L_{p}, L_{q}^{\prime}\right) \mid D\left(\omega ; L_{p}, L_{q}^{\prime}\right) \neq 0 \text { for all } \omega \in X_{0}\right\} .
$$

Obviously, $\hat{X}_{0}$ is a $G_{0}$ invariant subset of $Z$. We want to show that $\hat{X}_{0}$ is Stein. To do that, we need the following technical lemma in complex analysis, the proof provided in [Gu, p. 137] carries over verbatim.

Lemma 2.5. Let $V$ be a holomorphic variety and $U_{j}, j \in J$, is an arbitrary collection of Stein open subsets. Suppose $U:=\cap_{j} U_{j}$ is non-empty open, then $U$ is also a Stein variety.

We now have:

Lemma 2.6. The space $\hat{X}_{0}$ is a Stein variety.

Proof. We would like to apply Lemma 2.5. Let $V=Z$. For each $\omega \in X$, define $U_{\omega}=\left\{\left(L_{p}, L_{q}^{\prime}\right) \in Z \mid D\left(\omega, L_{p}, L_{q}^{\prime}\right) \neq 0\right\}$. Then $\hat{X}_{0}=\cap_{\omega \in X_{0}} U_{\omega}$.

It can be verified directly that $L_{p}=l_{p}, L_{q}^{\prime}=l_{q}^{\prime}$ belongs to $\hat{X}_{0}$. The compactness of $X_{0}$ guarantees that $\hat{X}_{0}$ is open.

It remains to see that each $U_{\omega}$ is Stein. Observe that $Z$ is in fact a smooth affine variety. Viewed in this way, each $D_{\omega}\left(L_{p}, L_{q}^{\prime}\right):=D\left(\omega ; L_{p}, L_{q}^{\prime}\right)$ can be viewed as a regular function on $Z$. The set $U_{\omega}$ is the complement of the 
closed set defined by $D_{\omega}=0$, and is thus a principal open set, hence also affine, and therefore Stein.

Lemma 2.7. The space $\hat{X}_{0}$ contains $Z_{0}$.

Proof. The space $\hat{X}_{0}$ is clearly $G_{0}$ invariant and contains the base point of $Z_{0}$ where $L_{p}=l_{p}, L_{q}^{\prime}=l_{q}^{\prime}$. However, $G_{0}$ acts transitively on $Z_{0}$.

Remark 2.8. It can be verified directly that $\hat{X}_{0}$ is connected except when $p=q$, and the connected component containing $Z_{0}$ is the space of linear cycles associated any flag domains. Clearly, this points to a close connection with the work in $[\mathbf{W o 1}],[\mathbf{W o 2}],[\mathbf{W o 3}],[\mathbf{W W}]$. It also relates to a recent work by Wolf and Zierau that the space of linear cycles have the structure $G_{0} / K_{0} \times \overline{G_{0} / K_{0}}[\mathbf{W Z}]$. See [BGW] for further details.

\section{Knapp-Wallach Construction.}

We briefly summarize Knapp-Wallach construction in the case $G_{0}=\mathrm{SU}(p, q)$.

The compact Cartan subgroup of $G_{0}$ is

$$
T_{0}=\left\{g=\operatorname{diag}\left(e^{i \theta_{1}}, \ldots, e^{i \theta_{n}}\right) \mid \sum_{j=1}^{n} \theta_{j}=0\right\},
$$

and we think of $i \mathfrak{t}_{0}=\left\{\left(\theta_{1}, \ldots, \theta_{n}\right) \mid \sum_{i=1}^{n} \theta_{i}=0\right\}$ as $\mathbb{R}^{n}$ in the obvious way.

If we denote by $f_{j} \in\left(\mathbb{R}^{n}\right)^{*}, j=1, \ldots n$, the elements of the dual basis of the standard basis $e_{j}$ of $\mathbb{R}^{n}$, then the roots of $\mathfrak{t}$ in $\mathfrak{g}$ correspond to the set

$$
\Delta(\mathfrak{g}, \mathfrak{t})=\left\{f_{i}-f_{j} \mid i \neq j ; 1 \leq i, j \leq n\right\} .
$$

The Cartan involution $\theta$ is defined by $\theta(X)=-X^{*}$ for $X \in \mathfrak{g}_{0}$. Thus we have $\mathfrak{g}=\mathfrak{k} \oplus \mathfrak{p}$. The set of compact imaginary roots of $\mathfrak{t}$ in $\mathfrak{g}$ is given by

$$
\Delta(\mathfrak{k}, \mathfrak{t})=\left\{f_{i}-f_{j} \mid 1 \leq i, j \leq p\right\} \cup\left\{f_{k}-f_{m} \mid p+1 \leq k, m \leq p+q\right\}
$$

and

$$
\Delta(\mathfrak{p}, \mathfrak{t})=\left\{ \pm\left(f_{i}-f_{p+j}\right) \mid 1 \leq i \leq p, 1 \leq j \leq q\right\}
$$

is the set of noncompact roots of $\mathfrak{t}$ in $\mathfrak{g}$.

Once a positive system $\Delta^{+}(\mathfrak{k}, \mathfrak{t})$ is fixed, the Knapp-Wallach construction starts with a triple $\left(\mathfrak{t}, \Delta^{+}(\mathfrak{g}, \mathfrak{t}), \lambda\right)$, where the positive system is chosen to be compatible with $\Delta^{+}(\mathfrak{k}, \mathfrak{t})$ and $\lambda \in \hat{K}_{0}$ is a dominant integral parameter so that $\lambda-\rho_{n}+\rho_{k}$ is regular dominant for $\Delta^{+}(\mathfrak{g}, \mathfrak{t})$.

Each triple determines uniquely a representation in the discrete series of $G_{0}([\mathbf{S c 2}])$. The Knapp-Wallach Szegö mapping relates a principal series with a discrete series. The details are as follows. 
First, to each triple $\left(\mathfrak{t}, \Delta^{+}(\mathfrak{g}, \mathfrak{t}), \lambda\right)$ is associated a new triple $\left(\hat{\mathfrak{t}} \oplus \mathfrak{a}, \Delta^{+}(\mathfrak{g}, \hat{\mathfrak{t}} \oplus\right.$ $\left.\mathfrak{a}),\left(\mu,\left.\lambda\right|_{\hat{\mathfrak{t}}}\right)\right)$, where $\hat{\mathfrak{t}} \oplus \mathfrak{a}$ a maximally split Cartan subalgebra of $\mathfrak{g}, \Delta^{+}(\mathfrak{g}, \hat{\mathfrak{t}} \oplus \mathfrak{a})$ a positive system for $\Delta(\mathfrak{g}, \hat{\mathfrak{t}} \oplus \mathfrak{a})$ with the property that if $\alpha \in \Delta^{+}$, then $\theta \alpha \notin$ $\Delta^{+}$, and $\mu \in \mathfrak{a}^{*}$. We would like to explain how this new triple determines a principal series representation of $G_{0}$.

To choose the maximally split Cartan subgroup, we have to start with a sequence of positive noncompact roots $\alpha_{1}, \ldots, \alpha_{q}$ which satisfies:

$$
\left\{\begin{array}{c}
\left\{\alpha_{i}\right\} \text { are strongly orthogonal (s.o.). } \\
\alpha_{j} \text { is simple in the subsystem of roots s.o. to }\left\{\alpha_{1}, \ldots, \alpha_{j-1}\right\} .
\end{array}\right.
$$

Such a sequence is called a strongly orthogonal sequence (s.o.s.). Let $\langle, \quad\rangle$ be the trace form, choose root vectors $E_{\alpha}$ so that

$$
\left\langle E_{\alpha}, E_{-\alpha}\right\rangle=\frac{2}{\langle\alpha, \alpha\rangle} .
$$

Define $H_{\alpha}$ to be the member of $\mathfrak{t}$ dual to $\alpha$ under $\langle$,$\rangle .$

The standard Cayley transform relative to the sequence $\alpha_{1}, \ldots, \alpha_{q}$ is $\mathbf{c}=$ $\operatorname{Ad}(u)$ with $u=u_{\alpha_{1}} \ldots u_{\alpha_{q}}$ and $u_{\alpha_{j}}=\exp \left(\frac{\pi}{4}\left(E_{\alpha_{j}}-E_{-\alpha_{j}}\right)\right)$. The maximally split Cartan subalgebra is $\mathfrak{a} \oplus \hat{\mathfrak{t}}=\mathbf{c t}=\Sigma\left(E_{\alpha_{j}}+E_{-\alpha_{j}}\right) \oplus \hat{\mathfrak{t}}$. Here $\hat{\mathfrak{t}}$ is the orthocomplement of $\Sigma_{1}^{q} i \mathbb{C} H_{\alpha_{j}} \in \mathfrak{t}$.

This takes care of the maximally split Cartan subgroup. Next, we need to explain the positive restricted root system. Since this is in turn pinned down by a positive root system of $\mathfrak{a} \oplus \hat{\mathfrak{t}}$, we will explain the choice of the latter.

The positive system $\Delta^{+}(\mathfrak{g}, \mathfrak{a} \oplus \mathfrak{t})$ is determined lexicographically with respect to the list $H_{\alpha_{1}} \ldots H_{\alpha_{q}}$. Indeed, a root $\mathbf{c} \beta$ is positive if $\left\langle\beta, \alpha_{1}\right\rangle<0$ or $\left\langle\beta, \alpha_{1}\right\rangle=0$ and $\left\langle\beta, \alpha_{2}\right\rangle<0$, etc. For roots $\mathbf{c} \beta$ supported on $\hat{\mathfrak{t}}$, then $\mathbf{c} \beta$ is positive if $\beta \in \Delta^{+}(\mathfrak{g}, \mathfrak{t})$.

Finally, we can take care of the parameter $\mu \in \mathfrak{a}^{*}$. It is defined by means of the equation

$$
\left(\mu-\rho_{a}\right)\left(E_{\alpha_{j}}+E_{-\alpha_{j}}\right)=\frac{-2\left\langle\lambda+n_{j} \alpha_{j}, \alpha_{j}\right\rangle}{\left\langle\alpha_{j}, \alpha_{j}\right\rangle}
$$

where $\rho_{a}$ denotes the half sum of the associated positive restricted roots. Here the integer $n_{j}$ is as in Equation (6.5b) on [KW, p. 178].

At this point, we have the principal series representation: The maximally split Cartan together with the positive system $\Delta^{+}(\mathfrak{g}, \mathfrak{a} \oplus \hat{\mathfrak{t}})$ determines a minimal parabolic subgroup $P_{0}=M_{0} A_{0} N_{0}$ of $G_{0}$. Let $\sigma$ be the irreducible representation of $M_{0}$ with highest weight $\left.\lambda\right|_{\hat{\mathfrak{t}}}$ (when $p=q, M_{0}$ is disconnected and one has to be a little more careful in defining $\sigma$ ). The principal series representation of $G_{0}$ is induced form $(\sigma, \mu)$. 
Finally, we can write down the Knapp-Wallach Szegö mapping.

If $(\tau, V)$ denotes the irreducible representation of $K_{0}$ with the highest weight $\lambda$, then Knapp-Wallach Szegö map is given by

$$
\mathcal{S} f(l)=\int_{K} \tau(k)[f(l k)] d k,
$$

where $f$ is a function on $G_{0}$ with the correct transformation property such that it represents an element of the principal series.

We would like to rewrite the mapping in a slightly different context.

If $P$ denotes the complexification of $P_{0}$, then $G_{0} / P_{0}$ is the unique closed orbit of the generalized flag manifold $Y=G / P$ (the same manifold as in Remark 2.3). The preimage of $G_{0} / P_{0}$, under the canonical fibration $X \longrightarrow$ $G / P$, is the unique closed orbit $X_{0}$ of $X$. Thus we can transfer geometrical data on $G_{0} / P_{0}$ to $X_{0}$. In particular, instead of a vector bundle on $G_{0} / P_{0}$, we can consider a related line bundle on $X_{0}$. In this context, the domain of the Szegö map is a space of certain smooth sections of an appropriate line bundle over $X_{0}$. Some of this is implicitly done in [KW, p. 180] when they rewrite their intertwining formula as a kernel operator.

More precisely, proceed as follows. The s.o.s. $\left\{\alpha_{1}, \ldots, \alpha_{q}\right\}$ determines a base point on the orbit $X_{0}$. Indeed, the set of roots $\Delta^{+}(\mathfrak{m}, \hat{\mathfrak{t}}) \cup\{\beta \in$ $\Delta(\mathfrak{g}, \mathfrak{t}) \mid\left\langle\beta, \alpha_{1}\right\rangle<0$, or $\left\langle\beta, \alpha_{1}\right\rangle=0$ and $\left\langle\beta, \alpha_{2}\right\rangle<0$, etc. $\}$ defines a Borel subalgebra $\mathfrak{b}^{\prime}=\mathfrak{t} \oplus \mathfrak{u}^{\prime}$. The base point in $X_{0}$ associated to $\alpha_{1}, \ldots, \alpha_{q}$ is given as the Borel subalgebra $\mathfrak{b}=\mathbf{c b}^{\prime}=\mathfrak{a} \oplus \hat{\mathfrak{t}} \oplus \mathfrak{u}$. We denote by $B$ the analytic subgroup of $G$ with Lie algebra $\mathfrak{b}$.

Let $\nu \in(\mathfrak{h}=\mathfrak{a} \oplus \hat{\mathfrak{t}})^{*}$ be so that $\left.\nu\right|_{\mathfrak{a}}=\mu+\rho_{a}$ and $\left.\nu\right|_{\hat{\mathfrak{t}}}=\left.\lambda\right|_{\hat{\mathfrak{t}}}$. This defines a line bundle $\mathcal{L}_{\nu}$ on $X$. Let $\Omega^{\text {top }}$ be the line bundle induced from $\wedge^{\text {top }}(\mathfrak{u} /(\mathfrak{u} \cap \mathfrak{m})$ ) (it can be interpreted as top holomorphic de Rham forms on $X$ "transversal" to the fibers of $X \longrightarrow G / P)$. Finally, let the $K$ module $(\tau, V)$ induce the vector bundle $V \rightarrow Z$. The Knapp-Wallach Szegö kernel can be viewed as a section of the bundle $\left(\mathcal{L}_{\nu}^{*} \otimes \Omega^{\text {top }}\right) \otimes V$ on $X_{0} \times Z_{0}$, where $Z_{0} \subseteq Z$ is the real form $G_{0} / K_{0}$ of $Z$.

When $x, l \in G_{0}$, let $x^{-1} l=\kappa a n \in K_{0} A_{0} N_{0}$ be the Iwasawa decomposition, then the Szegö kernel as given in $[\mathbf{K W}]$ can be written as

$$
S(x, l)=a\left(x^{-1} l\right)^{\mu-\rho} \tau\left(\kappa\left(x^{-1} l\right)\right) \phi_{\lambda} \times \operatorname{Ad}(l)(\Omega)
$$

where $\phi_{\lambda}$ denotes a highest weight vector of the irreducible representation $\tau$ and $\Omega$ is a non-zero element of $\Lambda^{\text {top }} \mathfrak{u} /(\mathfrak{u} \cap \mathfrak{m})$. In other words, suppose $f: G_{0} \rightarrow \mathbb{C}_{\nu}$ represents a section over $X_{0}$ which comes from the principal series, then for each $x, S(x, l) f(x)$ would represent the pullback of a top de Rham form on $G_{0} / P_{0}$, its integration over $G_{0} / P_{0}$ is $\mathcal{S} f(x)$. 
Remark 3.1. We will abuse terminology and say that $\mathcal{S} f(x)$ is the integration over $X_{0}$ of $S(x, l) f(l)$.

Remark 3.2. For $\mathrm{SU}(p, q)$, s.o.s. satisfying the Conditions 3.1 are not unique. Different choices of s.o.s. produce different choices of the base point in the unique closed $G_{0}$ orbit $X_{0}$ in $X$ and different line bundles over $X_{0}$. This may result in, as commented upon in [KW, p. 164], the realization of the same discrete series as quotients of different principal series. Sometimes we will choose some particular s.o.s. to carry out computations.

Each point $l \in G$ determines a point $l \cdot B \in X$, and each $x \in G$ can be thought as a point $x \cdot K \in Z$. In this context we want to obtain a meromorphic continuation of $S(x, l)$ in the most straightforward manner, simply as in Equation 3.2 but now let $x, l \in G$. Thus, we are led to consider the subset of $G$ whose elements $g$ are expressible as $\kappa a n \in K A N$. However, there is no complex Iwasawa decomposition because two things can potentially go wrong. First, not all $g \in G$ admits such a form. Second, $\kappa, a, n$ need not be unique. Theoretically, we can "remedy" these two defects as follows. We can, instead, define the Szegö kernel for those pairs of $(x, l)$ such that $g=x^{-1} l$ admits a complex Iwasawa decomposition, and then go on to prove that such a mapping continues meromorphically to all pairs. This should "remedy" the first defect. For the second defect, we can try to show that the integrality of $\lambda$ will guarantee that the formula will be well defined despite the fact that there may be several Iwasawa decompositions for the same $g$.

However, we choose to take a more pragmatic approach here. Since we are dealing with the special case of $G_{0}=\mathrm{SU}(p, q)$, we will naively extend the Szegö kernel formula complex analytically, and express it in terms of geometrical data, rather than group data. It will be apparent from the resulting formula that it is meromorphic and well defined.

\section{Knapp-Wallach Construction for $\mathrm{SU}(p, q)$ in Practice I.}

Suppose we have two rows of increasing numbers: $x_{i}$ in the first row, with $1 \leq x_{i} \leq p, y_{j}$ in the second row, with $p+1 \leq y_{j} \leq p+q$. An alignment of these two rows of numbers is called a double array. A double array looks like

$$
x_{1} \ldots x_{i_{1}} x_{i_{1}+1} \ldots x_{i_{2}} x_{y_{p+1}+1} \ldots y_{p+j_{1}} \ldots y_{p+j_{2}}
$$

We allow for the possibilities that $i_{1}=0$ (i.e., nothing precedes $y_{p_{1}}$ ) and $i_{1}+1=i_{2}$, etc.

If we let $x_{i}=i, 1 \leq i \leq p$ and $y_{m}=m, p+1 \leq m \leq p+q$, the associated double array is called a complete double array. The set of all positive systems 
$\Delta^{+}(\mathfrak{g}, \mathfrak{t})$ compatible with $\Delta_{K}^{+}$is in one-one correspondence with the set of all complete double arrays.

We can describe complete double arrays more precisely. A complete double array corresponds to a non-decreasing sequence of integers $0 \leq a_{1} \leq$ $\ldots \leq a_{q} \leq p$, as follows. The number $y_{p+i}$ in the second row must be squeezed in the space after the number $a_{i}$ and before $a_{i+1}$ in the first row. Alternatively, given such a sequence $a=\left(a_{i}\right)$, we have a related sequence $1 \leq b_{1}<\ldots<b_{q} \leq(p+q)$, by putting $b_{i}:=a_{i}+i$. The number $b_{i}$ refers to the position of $y_{p+i}$ in the double array picture. Thus, referring to the picture above, $b_{1}=i_{1}+1, b_{j_{1}}=i_{1}+j_{1}, b_{j_{1}+1}=i_{2}+1, \ldots$ As indicated in Remark 2.8, complete double arrays parametrize flag domains.

The simple roots are precisely $f_{i}-f_{j}$ whenever $x_{i}$ or $y_{i}$ is followed by $x_{j}$ or $y_{j}$. The non-compact simple roots are exactly $f_{i}-f_{j}$ for those $x_{i}$ followed by $y_{j}$ or those $y_{i}$ followed by $x_{j}$. Thus, in the diagram above, the simple roots are: $f_{1}-f_{2}, \ldots, f_{i_{1}-1}-f_{i_{1}}, f_{i_{1}}-f_{p+1}, f_{p+1}-f_{p+2}, \ldots, f_{p+j_{i}-1}-f_{p+j_{1}}, f_{p+j_{1}}-$ $f_{i_{1}+1}, \ldots$ The simple non-compact roots are: $f_{i_{1}}-f_{p+1}, f_{p+j_{1}}-f_{i_{1}+1}, f_{i_{2}}-$ $f_{p+j_{1}+1}, \ldots$

Remark 4.1. From elementary combinatorics it follows that there are $\frac{(p+q) !}{p ! q !}=\frac{\left|W_{G}\right|}{\left|W_{K}\right|}$ possible complete double arrays. Here $W_{G}, W_{K}$ denote the the respective Weyl groups. In general, there are $\frac{\left|W_{G}\right|}{\left|W_{K}\right|}$ different $G_{0}$-open orbits in $X$ (or, by duality, $K$-closed orbits in $X$ ) by [Wo1].

There are different possible choices of s.o.s. satisfying Conditions 3.1. For the sake of illustrating that the functions $D_{j}$ occur as singularities, we work with the choice picked out by the following algorithm. This algorithm also yields $n_{j}$.

\section{Algorithm 4.2.}

(1) Let $A$ be a complete double array. Start with the empty list as the initial list of s.o. roots. Set $j=1$.

(2) If the second row of $A$ is empty. Stop.

(3) Suppose the most left hand member of the top row of $A$ occurs to the left of the most left hand member $y_{p+r}$ of the second row. Let $x_{k}$ be the member in the first row that occurs immediately to the left of $y_{p+r}$. Append $f_{k}-f_{p+r}$ to the list. Let $n_{j}$ be the number of elements in the top row of $A$ to the right of $x_{k}$. Erase both $y_{p+r}, x_{k}$ from $A$. Reset $A$ to be the new double array and replace $j$ by $j+1$. Go to Step 2 .

(4) Suppose the most left hand element $x_{k}$ of the top row occurs to the right of the most left hand element of the second row. Let $y_{p+r}$ be the element in the second row immediately to the left of $x_{k}$. Append $f_{p+r}-f_{k}$ to the list. Let $n_{j}$ be the number of elements in the second 
row of $A$ to the right of $y_{p+r}$ Erase both $y_{p+r}, x_{k}$ from $A$. Reset $A$ to be the new double array and replace $j$ by $j+1$. Go to Step 2 .

Once the s.o.s. is selected, we can proceed as described above to determine a base point in the $G_{0}$-open orbit. The base point can be given in the form of a Borel subalgebra or in terms of an ordered basis $\left(v_{j}, 1 \leq j \leq p+q\right)$ of $\mathbb{C}^{p+q}$. For a given s.o.s., the $v_{j}$ are as follows:

(1) For $1 \leq i \leq q v_{i}=e_{l}+e_{r}$ where $f_{l}-f_{r}$ is the $\mathrm{i}$-th root in the s.o.s.

(2) For $q+1 \leq i \leq p v_{i}=e_{k_{i}}$ with $k_{i}<k_{i^{\prime}}$ if $i<i^{\prime}$. Here, the $f_{k_{i}}$ are so that none of the roots of the form $\pm\left(f_{k_{i}} \pm f_{s}\right), 1 \leq s \leq p+q$ are in the s.o.s.

(3) $v_{p+1}=e_{l}-e_{r}$ if $f_{l}-f_{r}$ is the last root in the s.o.s., etc.

(4) Until we have $v_{p+q}=e_{l}-e_{r}$ if $f_{l}-f_{r}$ is the first root in the s.o.s.

From $\left(v_{j}\right)$ we can form $\left(\omega_{0}\right)_{j}:=\wedge_{i=1}^{j} v_{i}$. It can be verified that $\left(\omega_{0}\right)_{j}$ is $N$ invariant. The group $A$ acts on them by an scalar. If $a \in A$, then $a \cdot\left(\omega_{0}\right)_{j}=a^{\alpha_{1}+\ldots+\alpha_{j}}\left(\omega_{0}\right)_{j}$ for $1 \leq j \leq q, a \cdot\left(\omega_{0}\right)_{j}=a^{\alpha_{1}+\ldots+\alpha_{q}}\left(\omega_{0}\right)_{j}$ for $q+1 \leq j \leq p$, and $a \cdot\left(\omega_{0}\right)_{j}=a^{\alpha_{1}+\ldots+\alpha_{q-k}}\left(\omega_{0}\right)_{j}$ for $j=p+k$.

\section{Knapp-Wallach Construction for $\mathrm{SU}(p, q)$ in Practice II.}

An element $l \in G$ is thought of as a point $l \cdot B$ in the flag manifold or equivalently as a point $\left(l \cdot\left(\omega_{0}\right)_{j}\right)=\left(\omega_{j}\right)$. An element $x \in G$ is identified with $x \cdot K$ or equivalently with the pair $\left(x \cdot l_{p}, x \cdot l_{q}^{\prime}\right)=\left(L_{p}, L_{q}^{\prime}\right)$. We want to rewrite the Szegö kernel as in Equation 3.2 in geometric terms. We will omit the term $\operatorname{Ad}(l)(\Omega)$ for we do not need it (see Remark 5.5).

We can assume that, under the trace form, $\lambda$ is the diagonal matrix

$$
\operatorname{diag}\left(\sum_{1}^{p} r_{i}, \sum_{2}^{p} r_{i}, \ldots, r_{p} ; \sum_{1}^{q} s_{i}, \sum_{2}^{q} s_{i}, \ldots, s_{q}\right),
$$

where $r_{i}, s_{j} \geq 0$ when $i \leq p-1, j \leq q-1$.

We find it convenient to think of $(\tau, V)$ as the unique subrepresentation of type $\tau$ in the following obvious representation of $K$.

$\left\{\left(\mathbb{C}^{p}\right)^{r_{1}} \otimes\left(\wedge^{2} \mathbb{C}^{p}\right)^{r_{2}} \otimes \ldots \otimes\left(\wedge^{p} \mathbb{C}^{p}\right)^{r_{p}}\right\} \otimes\left\{\left(\mathbb{C}^{q}\right)^{s_{1}} \otimes\left(\wedge^{2} \mathbb{C}^{q}\right)^{s_{2}} \otimes \ldots \otimes\left(\wedge^{q} \mathbb{C}^{q}\right)^{s_{q}}\right\}$.

Here $(\ldots)^{r_{i}}$ denotes the $r_{i}$-symmetric tensor. The representation $\tau$ is equivalent to the representation of $K$ acting on the cyclic span of $\left\{\left(e_{1}\right)^{r_{1}} \otimes\left(e_{1} \wedge\right.\right.$ $\left.\left.e_{2}\right)^{r_{2}} \otimes \ldots \otimes\left(e_{1} \wedge e_{2} \wedge \ldots \wedge e_{p}\right)^{r_{p}}\right\} \otimes\left\{\left(e_{p+1}\right)^{s_{1}} \otimes\left(e_{p+1} \wedge e_{p+2}\right)^{s_{2}} \otimes \ldots \otimes\left(e_{p+1} \wedge\right.\right.$ $\left.\left.\ldots \wedge e_{p+q}\right)^{s_{q}}\right\}$ (a highest weight vector) under $K$.

Call this bigger representation $(\tau, W)$. Notice that it admits an obvious extension to a representation of $G$. Hence, the obvious homogeneous bundle $G \times{ }_{K} W \rightarrow G / K$ is a trivial bundle (but non-trivial as a homogeneous 
bundle!): Take a basis $\left(w_{i}\right)$ for $W$, then the sections $g \mapsto\left(g, g^{-1} \cdot w_{i}\right) / \backsim$ forms a pointwise basis, here $\left(g, g^{-1} \cdot w_{i}\right) / \backsim$ means the equivalent class of $\left(g, g^{-1} \cdot w_{i}\right)$ under the usual equivalence relation. We will trivialize the bundle $G \times{ }_{K} W$ in such a manner.

It should now become obvious why we would like to embed $V$ into the larger space $W$. For each $l$, we can view $S(, l)$ as a function $S(, l): G \rightarrow W$ coming from a section of $G \times{ }_{K} W$. Under the trivialization explained above, we can view $S(, l)$ as a vector-valued function $G / K \rightarrow W: S(x \cdot K, l)=$ $x \cdot S(x, l)$. From now on we will understand $S(x, l)$ in this way.

Here is the general structure of the Szegö kernel, no matter how the s.o.s. is found.

Theorem 5.1. The Szegö kernel is a product of meromorphic functions of the form $\prod_{k=0}^{q} D_{j}\left(\omega_{j} ; L_{p}, L_{q}^{\prime}\right)^{n_{j}}$, where $n_{j} \in \mathbb{Z}$, with a suitable holomorphic section.

Remark 5.2. We will prove Theorem 5.1 in the next section. It should be noted that the proof contains a general algorithm which tells us how to write down the formula for the Szegö kernel in general. The actual formula depends crucially on the actual choice of s.o.s. Since a general description of s.o.s. is combinatorially complicated, we are unable to give a "one-line" formula for the Szegö kernel. Further, in the formula resulted, the holomorphic section may share common factors with the meromorphic function. Hence, we cannot be sure of the precise singularities and their degrees.

We have the following immediate corollary.

Corollary 5.3. The singularities of a Szegö kernel is always a union of the zero set of some of the $D_{k}$, i.e., $\cup_{k \in A}\left\{\left(\omega_{j} ; L_{p}, L_{q}^{\prime}\right) \mid D_{k}\left(\omega_{j} ; L_{p}, L_{q}^{\prime}\right)=0\right\}$ for some subset $A \subseteq\{0, \ldots, q\}$.

We have the following remarkable consequence straightaway.

Theorem 5.4. When $\lambda$ is far from the wall, in the sense of $[\mathbf{K W}]$, any solution of the Schmid equation admits a holomorphic extension to $\hat{X}_{0}$.

Proof. By [KW, Corollary 9.6], the image of the real version of the KnappWallach Szegö mapping is exactly the solution space.

Now the Szegö mapping is the integration over $X_{0}$ of the Szegö kernel (recall Remark 3.1). Since the only singularity of the kernel is always outside $\hat{X}_{0}$, the Szegö mapping sends a section over $X_{0}$ to a section defined for any point in $\hat{X}_{0}$ and the resulting section over $\hat{X}_{0}$ is clearly holomorphic. Its restriction to $Z_{0}$ is clearly what is given by the real version of the Szegö mapping. 
Remark 5.5. We have been ignoring the term $\operatorname{Ad}(l) \omega$ in Equation 3.2 for the Szegö kernel. This is because $\operatorname{Ad}(l) \Omega$, when restricted to $\hat{X}_{0}$, is essentially the pullback of a $K_{0}$ invariant top form on $G_{0} / P_{0}$ and would not contribute any singularity.

For the rest of this section, we assume that we choose the s.o.s. by Algorithm 4.2 .

It is convenient to introduce the notation $\Omega_{k}:=\left(\omega_{k} \sqcup L_{q}^{\prime}\right) \sqcap L_{p}$ in order to state the following results.

For the next theorem, assume we are dealing with the following special sort of complete double array:

$$
x_{1} \ldots x_{i_{1}} x_{i_{1}+1} \ldots x_{i_{2}} \ldots \quad x_{i_{q-1}+1} \ldots x_{i_{q}} x_{y_{p+q}} \ldots x_{p}
$$

where $1 \leq i_{1}<\ldots<i_{q}$.

Theorem 5.6. If $x \cdot l_{p}=L_{p}, x \cdot l_{q}^{\prime}=L_{q}, l \cdot\left(\omega_{0}\right)_{j}=\omega_{j}$, then the Szegö kernel, expressed as $S(x \cdot K, l)$, is, up to a non-zero constant multiple, the product of the following meromorphic function

$$
\begin{aligned}
D_{q}\left((\omega) ; L_{p}, L_{q}^{\prime}\right)^{s_{q}-\left(r_{1}+\ldots+r_{p}\right)-\left(p-i_{q}\right)} \times D_{0}^{-\left(r_{i_{q}}+\ldots+r_{p-1}\right)-\left(p-i_{q}\right)} \\
\quad \times \prod_{\{k \mid 1 \leq k \leq q-1\}} D_{k}^{-\left(r_{i_{k}}+\ldots+r_{\left(i_{k+1}-1\right)}\right)-\left(i_{k+1}-i_{k}\right)}
\end{aligned}
$$

(when $i_{q}=p$ (in particular, if $p=q$ ), the factor $D_{0}$ should be interpreted as not being involved) with the following holomorphic section:

$$
\begin{gathered}
\left\{\bigotimes_{a=1}^{i_{1}-1}\left(\omega_{a+q} \sqcap L_{p}\right)^{r_{a}} \otimes \bigotimes_{a=i_{1}}^{i_{2}-1}\left(\left(\omega_{a+q-1} \sqcap L_{p}\right) \sqcup \Omega_{1}\right)^{r_{a}} \otimes \ldots \otimes\right. \\
\bigotimes_{\substack{a=i_{k} \\
i_{q}-1}}^{\sum_{k+1}-1}\left(\left(\omega_{a+q-k} \sqcap L_{p}\right) \sqcup \Omega_{k}\right)^{r_{a}} \otimes \ldots \otimes \\
\bigotimes_{a=i_{q-1}}\left(\left(\omega_{a+q-(q-1)} \sqcap L_{p}\right) \sqcup \Omega_{q-1}\right)^{r_{a}} \otimes \\
\left.\bigotimes_{a=i_{q}}^{p-1} \Omega_{a}^{r_{a}} \otimes L_{p}^{r_{p}}\right\} \\
\otimes\left\{\bigotimes_{a=1}^{q-1}\left(\left(\omega_{a} \sqcup L_{p}\right) \sqcap L_{q}^{\prime}\right)^{s_{a}} \otimes\left(L_{q}^{\prime}\right)^{s_{q}}\right\} .
\end{gathered}
$$

Remark 5.7. Suppose $i_{k}=k$ for $k \leq j$ but $i_{j+1} \neq j+1$. Observe in that case $i_{k}=i_{k+1}-1$ for $k \leq j-1$ and that $\omega_{i_{k}-k+q} \sqcap L_{p} \doteq D_{q}$ for $k \leq j$. 
Piece these together, we can rewrite the formula in Theorem 5.6 as follows. The meromorphic function stays the same except that the exponent of $D_{q}$ becomes $s_{q}-\left(r_{j+1}+\ldots+r_{p}\right)-\left(p-i_{q}\right)$.

The section becomes

$$
\begin{gathered}
\left\{\bigotimes_{a=1}^{j}\left(\Omega_{a}\right)^{r_{a}} \otimes \bigotimes_{a=j+1}^{i_{j+1}-1}\left(\left(\omega_{a+q-j} \sqcap L_{p}\right) \sqcup \Omega_{j}\right)^{r_{a}} \otimes\right. \\
\bigotimes_{k=j+1}^{q-1} \bigotimes_{a=i_{k}}^{i_{k+1}-1}\left(\left(\omega_{a+q-k} \sqcap L_{p}\right) \sqcup \Omega_{k}\right)^{r_{a}} \otimes \ldots \otimes \\
\left.\bigotimes_{a=i_{q}}^{p-1} \Omega_{a}^{r_{a}} \otimes L_{p}^{r_{p}}\right\} \\
\otimes\left\{\bigotimes_{a=1}^{q-1}\left(\left(\omega_{a} \sqcup L_{p}\right) \sqcap L_{q}^{\prime}\right)^{s_{a}} \otimes\left(L_{q}^{\prime}\right)^{s_{q}}\right\} .
\end{gathered}
$$

Proposition 5.8. Keep the notations in Theorem 5.6. In the case of antiholomorphic discrete series:

$$
{ }_{y_{p+1} \ldots y_{p+q}}^{x_{1} \ldots x_{p}}
$$

the Szegö kernel, expressed as $S(x \cdot K, l)$, is the product of the meromorphic function $D_{0}^{r_{p}-\left(s_{1}+\ldots+s_{q}\right)}$ with the holomorphic section

$$
\left\{\bigotimes_{a=1}^{p-1}\left(\Omega_{a}\right)^{r_{a}} \otimes\left(L_{p}\right)^{r_{p}}\right\} \otimes\left\{\bigotimes_{a=1}^{q-1}\left(\omega_{a+p} \sqcap L_{q}^{\prime}\right)^{s_{a}} \otimes\left(L_{q}^{\prime}\right)^{s_{q}}\right\} .
$$

From these follows this corollary:

Corollary 5.9. Assume $\left\langle\lambda-\delta_{n}+\delta_{k}, \alpha\right\rangle \geq 0$ for all $\alpha \in \triangle^{+}(\mathfrak{g}, \mathfrak{t})$. Each of the determinant functions $D_{j}$ occurs as part of the singularity of the Szegö kernel associated with a suitable discrete series.

We will prove these results in the next section.

\section{Proofs.}

The following simple but crucial observation follows from a simple piece of linear algebraic calculation.

$$
\begin{aligned}
{\left[\omega_{p} \sqcap L_{q}^{\prime}\right] } & =a\left(x^{-1} l\right)^{\alpha_{1}+\ldots+\alpha_{q}} \Delta_{1} \\
{\left[\omega_{q} \sqcap L_{p}\right] } & =a\left(x^{-1} l\right)^{\alpha_{1}+\ldots+\alpha_{q}} \Delta_{2} \\
{\left[\left(\omega_{p+q-k} \sqcap L_{p}\right) \sqcup \omega_{k} \sqcup L_{q}^{\prime}\right] } & =a\left(x^{-1} l\right)^{2\left(\alpha_{1}+\ldots+\alpha_{k}\right)}
\end{aligned}
$$


for $1 \leq k \leq q-1$. We view $\kappa\left(x^{-1} l\right)$ as a diagonal block matrix in $\operatorname{GL}(p) \times$ $\mathrm{GL}(q) \subseteq \mathrm{GL}(p+q)$ and $\Delta_{1}$ refers to the determinant of the submatrix of $\kappa\left(x^{-1} l\right)$ in $\operatorname{GL}(p), \Delta_{2}$ to the determinant of the submatrix in $\operatorname{GL}(q)$.

Proof of Theorem 5.1. It will be very convenient to introduce the following notations. For $1 \leq k \leq p-1, U_{k}:=x \cdot \kappa \cdot\left(e_{1} \wedge \ldots \wedge e_{k}\right)$, for $1 \leq k \leq q-1$, $V_{k}:=x \cdot \kappa \cdot\left(e_{p+1} \wedge \ldots \wedge e_{p+k}\right)$.

Let $\left(\alpha_{i} ; 1 \leq i \leq q\right)$ be the s.o.s. Let $\alpha_{k}=f_{a_{k}}-f_{p+b_{k}}$ or $\alpha_{k}=f_{p+b_{k}}-f_{a_{k}}$. Therefore $\left\{b_{i}\right\}=\{1, \ldots, q\}$ and $\left\{a_{i}\right\} \subseteq\{1, \ldots, p\}$. Let $c_{1}<c_{2} \ldots<c_{p-q}$ be such that $\left\{c_{l}\right\} \cup\left\{a_{i}\right\}=\{1, \ldots, p\}$.

Let $\tilde{D}_{k}=D_{k}$ for $1 \leq k \leq q-1$ but $\tilde{D}_{q}=D_{0} D_{q}$.

Observe that, if $a_{j}=J$, then

$$
x \cdot \kappa \cdot e_{J}=\tilde{D}_{j}^{-1} a\left(x^{-1} l\right)^{\alpha_{j}}\left(\omega_{p+q-j+1} \sqcap\left(\omega_{j} \sqcup L_{q}^{\prime}\right) \sqcap L_{p}\right) .
$$

Also, observe that

$$
\Lambda_{m=1}^{i}\left(x \cdot \kappa \cdot e_{c_{m}}\right)=D_{q}^{-1}\left(\omega_{q+i} \sqcap L_{p}\right) .
$$

From now on, assume $1 \leq a_{j(1)}<\ldots<a_{j(q)} \leq p$ and $b_{i(k)}=k$ for $1 \leq k \leq q$.

For each $1 \leq m \leq p-1$, let $\left\{a_{1}, \ldots, a_{q}\right\} \cap\{1, \ldots, m\}=\left\{a_{j(1)}, \ldots, a_{j(M)}\right\}$ and $\left\{a_{j(1)}, \ldots, a_{j(M)}\right\} \cup\left\{c_{1}, \ldots, c_{N}\right\}=\{1, \ldots, m\}$.

Let $\widehat{\Omega_{k}}:=\omega_{p+q-k+1} \sqcap\left(\omega_{k} \sqcup L_{q}^{\prime}\right) \sqcap L_{p}$, and we define

$$
U_{m}^{\prime}:=\left(\omega_{q+N} \sqcap L_{p}\right) \sqcup \widehat{\Omega_{j(1)}} \sqcup \ldots \sqcup \widehat{\Omega_{j(M)}} .
$$

It can be verified that

$$
U_{m} \doteq \Pi_{k=1}^{M}\left(\tilde{D}_{j(k)}\right)^{-1} \times D_{q}^{-1} \times \Pi_{k=1}^{M} a\left(x^{-1} l\right)^{\alpha_{j(k)}} U_{m}^{\prime} .
$$

Similarly, let $\Omega_{k}^{\prime}:=\omega_{p+q-k+1} \sqcap\left(\omega_{k} \sqcup L_{p}\right) \sqcap L_{q}^{\prime}$, we can define

$$
V_{n}^{\prime}:=\Omega_{i(1)}^{\prime} \sqcup \ldots \sqcup \Omega_{i(n)}^{\prime},
$$

then

$$
V_{n} \doteq \Pi_{k=1}^{n}\left(\tilde{D}_{i(k)}\right)^{-1} \times \Pi_{k=1}^{n} a\left(x^{-1} l\right)^{\alpha_{i(k)}} V_{n}^{\prime} .
$$

Now let $a\left(x^{-1} l\right)^{\alpha_{i}}$ be $e^{t_{i}}$, then $a\left(x^{-1} l\right)^{\mu-\rho}=\Pi_{k=1}^{q}\left(e^{t_{k}}\right)^{ \pm\left(R_{k}-S_{k}\right)-2 n_{k}}$, where $R_{k}=\sum_{i=a_{k}}^{p} r_{i}, S_{k}=\sum_{i=b_{k}}^{q} s_{i}$ and the sign is negative if $\alpha_{k}=f_{a_{k}}-f_{p+b_{k}}$ and positive if $\alpha_{k}=f_{p+b_{k}}-f_{a_{k}}$.

Group these together, we conclude that

$$
a\left(x^{-1} l\right)^{\mu-\rho} x \cdot \kappa \cdot \phi_{\lambda}=\Pi_{k=1}^{q} D_{k}^{-\left(R_{k}+S_{k}\right)} \times \Pi_{k=1}^{q}\left(e^{2 t_{k}}\right)^{M_{k}}
$$




$$
\begin{aligned}
& \times D_{0}^{-\left(R_{q}+S_{q}\right)} \times D_{q}^{-\sum_{i=1}^{p} r_{i}} \\
& \bigotimes_{k=1}^{p}\left(U_{k}^{\prime}\right)^{r_{k}} \otimes \bigotimes_{k=1}^{q}\left(V_{k}^{\prime}\right)^{s_{k}},
\end{aligned}
$$

where $M_{k}=S_{k}-n_{k}$ or $R_{k}-n_{k}$ depending on whether $\alpha_{k}$ is $f_{a_{k}}-f_{b_{k}}$ or $f_{b_{k}}-f_{a_{k}}$. done.

Recall that $e^{2 t_{k}}=\frac{D_{k}}{D_{k-1}}$ for $2 \leq k \leq q-1, e^{2 t_{1}}=D_{1}, e^{2 t_{q}}=\frac{D_{0} D_{q}}{D_{q-1}}$, we are

The remaining results to be proved are all stated under the assumption that we are using Algorithm 4.2 to pick the s.o.s.

Proof of Theorem 5.6. It is a simple exercise of linear algebra to verify that for $a<i_{1}$,

$$
\omega_{a+q} \sqcap L_{p} \doteq D_{q} x \cdot \kappa \cdot\left(e_{1} \wedge \ldots \wedge e_{a}\right),
$$

for $i_{k} \leq a<i_{k+1}, 1 \leq k \leq q-1$, we have

$$
\left(\omega_{a+q-k} \sqcap L_{p}\right) \sqcup \Omega_{k} \doteq D_{q} a\left(x^{-1} l\right)^{\left(\alpha_{1}+\ldots+\alpha_{k}\right)} x \cdot \kappa \cdot\left(e_{1} \wedge \ldots \wedge e_{a}\right),
$$

for $i_{q} \leq a$ we have

$$
\Omega_{a} \doteq a\left(x^{-1} l\right)^{\left(\alpha_{1}+\ldots+\alpha_{q}\right)} x \cdot \kappa \cdot\left(e_{1} \wedge \ldots \wedge e_{a}\right),
$$

whereas for $1 \leq b \leq q-1$ we have

$$
\left(\omega_{b} \sqcup L_{p}\right) \sqcap L_{q}^{\prime} \doteq a\left(x^{-1} l\right)^{\left(\alpha_{1}+\ldots+\alpha_{b}\right)} x \cdot \kappa \cdot\left(e_{p+1} \wedge \ldots \wedge e_{p+b}\right) .
$$

Finally, $\quad a\left(x^{-1} l\right)^{\mu-\rho} \doteq \Pi_{k=1}^{q}\left(e^{t_{k}}\right)^{\left(s_{k}+\ldots+s_{q}\right)-\left(r_{i_{k}}+\ldots+r_{p}\right)-2\left(p-i_{k}\right)}, \quad$ where $a\left(x^{-1} l\right)^{\alpha_{k}}=e^{t_{k}}$.

Piece these up together and simplify, we reach the formula.

Proof of Proposition 5.8. It can be verified that for $1 \leq a \leq q$,

$$
x \cdot \kappa \cdot\left(e_{1} \wedge \ldots \wedge e_{a}\right) \doteq a\left(x^{-1} l\right)^{-\left(\alpha_{1}+\ldots+\alpha_{a}\right)} \Omega_{a},
$$

for $q+1 \leq a \leq p$,

$$
x \cdot \kappa \cdot\left(e_{1} \wedge \ldots \wedge e_{a}\right) \doteq a\left(x^{-1} l\right)^{-\left(\alpha_{1}+\ldots+\alpha_{q}\right)} \Omega_{a},
$$

for $1 \leq b \leq q$,

$$
x \cdot \kappa \cdot\left(e_{p+1} \wedge \ldots \wedge e_{p+b}\right) \doteq a\left(x^{-1} l\right)^{-\left(\alpha_{1}+\ldots+\alpha_{q-b}\right)} \Delta_{1}^{-1}\left(\omega_{p+b} \sqcap L_{q}^{\prime}\right) .
$$


It is simple to check that $a^{\mu-\rho} \doteq \Pi_{k=1}^{q}\left(e^{t_{k}}\right)^{\left(r_{k}+\ldots+r_{p}\right)-\left(s_{q-k+1}+\ldots+s_{q}\right)}$. Piece these together, we are done.

Proof of Corollary 5.9. First of all, we would like to see that for both the case when $i_{j}=j, 1 \leq j \leq q$, in Remark 5.7 and the case of Proposition 5.8, the holomorphic section does not vanish identically on the set $\left\{\left(\omega ; L_{p}, L_{q}^{\prime}\right) \mid D_{j}\left(\omega ; L_{p}, L_{q}^{\prime}\right)=0\right\}$ for $0 \leq j \leq q$. It suffices to find, for each $0 \leq j \leq q$, a point of $X$ represented by $\omega_{0}$ such that $D_{j}\left(\omega_{0} ; l_{p}, l_{q}^{\prime}\right)=$ 0 but the holomorphic section does not vanish on $\left(\omega_{0} ; l_{p}, l_{q}^{\prime}\right)$. The following choice of base points $\omega_{0}(j)$ turns out to be what we need, as a simple but tedious linear algebraic computation reveals. We will simply pin down the base point by choosing a basis. For $1 \leq j \leq q-1$, it is $(e_{1}+e_{p+1}, \ldots, e_{q}+e_{p+q}, \overbrace{e_{q+1}, \ldots, e_{p}}, e_{q}-e_{p+q}, \ldots, e_{j+2}-e_{p+j+2}, e_{j}-$ $\left.e_{p+j}, e_{j+1}-e_{p+j+1}, e_{j-1}-e_{p+j-1}, \ldots, e_{1}-e_{p+1}\right)$. For $j=0$ it is $\left(e_{1}+\right.$ $e_{p+1}, \ldots, \overbrace{e_{q}+e_{p+q}, e_{q+1}, \ldots, e_{p-1}}, e_{p+q}, e_{p}, e_{q-1}-e_{p+q-1}, \ldots, e_{1}-e_{p+1})$. For $j=q$, it is $(e_{1}+e_{p+1}, \ldots, e_{q-1}+e_{p+q-1}, e_{q}, \overbrace{e_{q+1}+e_{p+q}, e_{q+2}, \ldots, e_{p}}, e_{q}-$ $\left.e_{p+q}, \ldots, e_{1}-e_{p+1}\right)$. (When $p=q$, the portion under the overbraces are skipped.)

To complete the proof, we need to see that the exponents of various $D_{j}$ involved in the formulae in Remark 5.7 and Proposition 5.8 are strictly negative. It turns out to be an immediate consequence of the inequality $\langle\lambda-$ $\left.\rho_{n}+\rho_{k}, \alpha\right\rangle \geq 0$. We will demonstrate these only for the (more complicated) case of Remark 5.7, and omit the easier (and similar) case of Proposition 5.8.

Under the trace form, $\rho_{k}$ is the diagonal matrix

$$
\frac{1}{2} \operatorname{diag}((p-1),(p-3), \ldots,-(p-1) ;(q-1),(q-3), \ldots,-(q-1)),
$$

and $\rho_{n}$ is the diagonal matrix

$$
\operatorname{diag}\left(a_{1}, \ldots, a_{p} ; p-2 i_{1}, p-2 i_{2}, \ldots, p-2 i_{q}\right),
$$

where $a_{k}=q-2 j+2$ iff $i_{j-1}+1 \leq k \leq i_{j}$, for $j=1, \ldots, q+1$ and with the convention that $i_{0}=0, i_{q+1}=p$.

It can be verified that the exponents of $D_{r}$ is $-\left(\left\langle\lambda-\rho_{n}+\rho_{k}, \beta_{r}\right\rangle+1\right)$, with $\beta_{r}$ defined as below. For $r=0, \beta$ is the root $f_{i_{q}}-f_{p}$ (when $i_{q}<p$ ), for $r=q$ and with $j$ the same meaning as in Remark 5.7, $\beta_{q}=f_{j+1}-f_{p+q}$, for $1 \leq r \leq q-1, \beta_{r}=f_{i_{r}}-f_{i_{r+1}}$. As all $\beta_{r}$ are positive roots, we are done.

\section{References}

[AG] D.N. Akheizer and S.G. Gindikin, On the Stein Extensions of Real Symmetric Spaces, Math. Ann., 286 (1990), 1-12.

[BGW] L. Barchini, S.G. Gindikin and H.W. Wong, Determinant Functions and the Geometry of the Flag Manifold for SU(p,q), J. of Lie Theory, 6(2) (1996), 191-206. 
[Gi] S.G. Gindikin, The Holomorphic Cauchy-Szegö Kernel for Non-Holomorphic Representations of $\mathrm{SU}(2,1)$, Contemp. Math., 191 (1995), 75-82.

[GH] S.G. Gindikin and G.M. Henkin, Integral Geometry for $\bar{\partial}$-Cohomology in q-linear Concave Domains in $\mathbb{C P}^{n}$, Funk. Anal. Prilozh., 12(4) (1978), 6-23. (Engl. transl.: Func. Anal. Appl., 12 (1978), 247-261).

[Gu] R.C. Gunning, Introduction to Holomorphic Functions of Several Variables, Vol. III, Homological Theory, Wadsworth, Belmont, CA, 1990.

[Kn] A.W. Knapp, A Szegö Kernel for Discrete Series, Proc. Int. Congress Math., 2 (1974), 99-104.

[KW] A.W. Knapp and N.R. Wallach, Szegö Kernels Associated with Discrete Series, Inv. Math., 34 (1976), 163-200. See also A.W. Knapp and N.R. Wallach, Correction and Addition to Szegö Kernels Associated with Discrete Series, Inv. Math., 62 (1980), 341-346.

[Mar1] A. Martineau, Sur la topologie des espaces de fonctions holomorphes, Math. Ann., 163 (1966), 62-88.

[Mar2] , Equations différentielles d'ordre infini, Bull. Soc. Math. France, 95 (1967), 109-154.

[Sc1] W. Schmid, Homogeneous complex manifolds and representations of semisimple Lie groups, Dissertation, University of California, Berkeley, 1967. Reprinted in "Representation theory and harmonic analysis on semisimple Lie groups", pp. 223-286, Mathematical Surveys and Monographs, 31, Amer. Math. Soc., Providence, 1989.

[Sc2] S Some Properties of Square-Integrable Representations of Semisimple Lie Groups, Ann. Math., 102 (1975), 535-564.

[WW] R.O. Wells and J.A. Wolf, Poincaré series and automorphic cohomology on flag domains, Ann. Math., 105 (1977), 397-448.

[Wo1] J.A. Wolf, The Action of a Real Semisimple Group on a Compl Flag Manifold, I: Orbit Structure and Holomorphic Arc Components, Bull. Amer. Math. Soc., 75 (1969), 1121-1237.

[Wo2] - The Stein Condition for Cycle Spaces of Open Orbits on Complex Flag Manifolds, Ann. Math., 136 (1992), 541-555.

[Wo3] _ Exhaustion functions and cohomology vanishing theorem for open orbits on complex flag manifolds, Mathematical Research Letters, 2 (1995), 179-191.

[WZ] R. Zierau, personal communication.

Received September 22, 1995 and revised March 21, 1996. The second author was supported by NSF Grant DMS 9202049.

OKLAhoma State University

STILlWATER, OK 74078

Rutgers UNIVERSITY

NEW BRunswiCK, NJ 08903

E-mail address: gindikin@math.rutgers.edu

AND

The University of Hong Kong

Porkfulam RD., Hong Kong

E-mail address: hwong@submaths.hku.hk 